\title{
Quadrupedal locomotion on uneven terrain with sensorized feet
}

\section{Journal Article}

\section{Author(s):}

Valsecchi, Giorgio; Grandia, Ruben; $\underline{\text { Hutter, Marco (D) }}$

Publication date:

2020-04

Permanent link:

https://doi.org/10.3929/ethz-b-000392778

\section{Rights / license:}

In Copyright - Non-Commercial Use Permitted

\section{Originally published in:}

IEEE Robotics and Automation Letters 5(2), https://doi.org/10.1109/LRA.2020.2969160

\section{Funding acknowledgement:}

780883 - subTerranean Haptic INvestiGator (EC) 


\title{
Quadrupedal locomotion on uneven terrain with sensorized feet
}

\author{
Giorgio Valsecchi ${ }^{1}$, Ruben Grandia ${ }^{1}$, Marco Hutter ${ }^{1}$
}

\begin{abstract}
Sensing of the terrain shape is crucial for legged robots deployed in the real world since the knowledge of the local terrain inclination at the contact points allows for an optimized force distribution that minimizes the risk of slipping. In this paper, we present a reactive locomotion strategy for torque controllable quadruped robots based on sensorized feet. Since the present approach works without exteroceptive sensing, it is robust against degraded vision. Inertial and force/torque sensors implemented in specially designed feet with articulated passive ankle joints measure the local terrain inclination and interaction forces. The proposed controller exploits the contact null-space in order to minimize the tangential forces to prevent slippage even in case of extreme contact conditions. We experimentally tested the proposed method in laboratory experiments and validated the approach with the quadrupedal robot ANYmal.
\end{abstract}

Index Terms-Mechanism Design; Legged Robots; Motion Control

\section{INTRODUCTION}

$\mathbf{L}$ EGGED robots have superior potential to overcome rough terrain compared to wheeled or tracked systems as they can discretely select individual footholds. However, maintaining stable footholds and avoiding slippage are still major challenges, as they imply a good knowledge of the local interaction between the foot and the environment. In particular, in order to avoid slippage, the ground reaction forces at all contact points must remain within the friction cone. It is, therefore, necessary to estimate the local orientation of the ground in order to shape the ground reaction force distribution appropriately.

Additionally, depending on the number of feet in contact, the amount of contact forces exceeds the number of floating base degrees of freedom to be controlled. This means that there is a null-space available that makes it possible to optimize for secondary objectives for the contact forces, such as minimizing torques, minimizing forces, or as done in this work, minimize the risk of slipping.

Minimizing the risk of slipping is achieved by aligning ground reaction forces with local surface normal directions. This can be achieved by reactive strategies which rely on proprioception and approximations of the terrain or by proactive strategies which require knowledge of the environment [1], available a priori or acquired with adequate sensors.

Manuscript received September, 10, 2019; Revised December, 12, 2019; Accepted January, 12, 2020.

This paper was recommended for publication by Editor Paolo Rocco upon evaluation of the Associate Editor and Reviewers' comments. This work has been supported by the European Union's Horizon 2020 research and innovation program under grant agreement No 780883. This work has been conducted as part of ANYmal Research, a community to advance legged robotics.

1 All the authors are with the Robotic Systems Laboratory, ETH Zurich, Zurich 8092, Switzerland vgiorgiodethz.ch rgrandia@ethz.ch mahutter@ethz.ch

Digital Object Identifier (DOI): see top of this page.
Simple reactive approaches can only redistribute the ground reaction force among the existing contacts, according to their knowledge of the ground shape, while proactive approaches have the option of planning the footholds to exploit the features of the terrain better. However, in real-world situations, the sensing capabilities of the robot might be impaired, and it is not always possible to build an adequate $3 \mathrm{D}$ model of the terrain. A multitude of reasons can impose such limitations: partially flooded terrains, environments with dust, fog, and smoke, vegetation occluding the field of view, mud, failing vision sensors. Similar conditions can be found in underground tunnels, mines, collapsed or burning buildings, forests, scenarios where is highly desirable to deploy robots. Moreover, even if it is possible to know the terrain geometry before stepping, the ground might shift, or small measurement inaccuracies can cause the real foothold to be different from the planned.

This work focuses on improving robot locomotion in the scenarios mentioned above. The approach has been implemented utilizing specially designed passive adaptive sensorized feet and a hierarchical whole-body control algorithm that optimizes the contact force distribution on the legged robotic platform ANYmal. The additional information provided by the feet allows the reactive strategy to achieve a better distribution of the ground reaction forces.

\section{A. Related work}

Sensorized feet have been developed before in robotics, serving different purposes in quadrupeds and bipeds. Most biped robots use feet with actuated dorsiflexion/plantarflexion and inversion/eversion (necessary for static stability) as the Honda ASIMO robot [2], Lola [3], KHR-3 [4] and WALKMAN [5] equipped with 6-axes force/torque transducers. Quadruped robots on the other hand typically have ball-shaped feet without sensors [6] [7] [8] [9], while extended and adaptive feet with articulated ankles are less common [10] [11]. Sensorized feet for quadruped have also been experimented, based on different sensing principles, such as strain gauge force sensor [12], sensitive skin [13] [14] [15], optical force sensors [16] and vibrissae [17].

For quadruped robots, the terrain shape sensing, necessary to minimize the risk of slipping, has been addressed both with reactive and proactive strategies. [18], [19] and [20] assumed complete knowledge of the environment to have quadruped robots walking over constant slopes or steep grooves. Surface normal estimation by fitting planes over contact points has been used in [21], [22] and [8]. [16] used additional sensor located in the foot to estimate local normal vectors. [23] and [24] used external motion capture and high-resolution scans of the terrain. Online exteroceptive range sensor are used in [25]. [26] proposed a more extended approach, which mixes 
reactive and proactive strategies while taking into account the degradation of visual feedback.

\section{B. Contribution}

We present a novel sensorized foot characterized by an articulated passive ankle with 2 degrees of freedom, sixaxis force/torque sensors and IMUs, integrated into a robust modular unit interfaceable with ANYmal. We propose an algorithm for estimating the foot pose using the data from the integrated IMUs. We present an integration of the tactile feedback in the hierarchical whole-body control approach from [27], with the capability of optimizing the ground reaction force. The utilization of the sensed local surface normals is two-fold: 1) the friction cone is aligned with the local surface to put a hard constraint on the contact force direction, and 2) the risk of slippage is minimized in the null-space of the motion control tasks. We tested the improved sensing capabilities of ANYmal. We performed the validation of the proposed controller through experiments on selected terrains. Field tests have been conducted to establish the effect on the locomotion of the proposed approach.

\section{METHOD}

\section{A. sensorized foot}

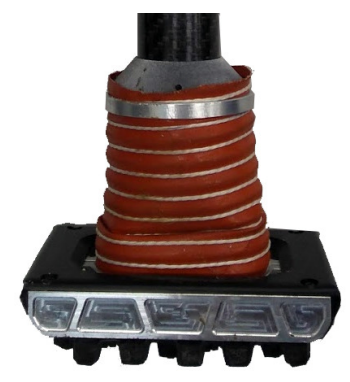

(a) Side view

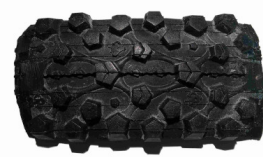

(b) Bottom view
Fig. 1: Side and bottom views of the newly developed foot.

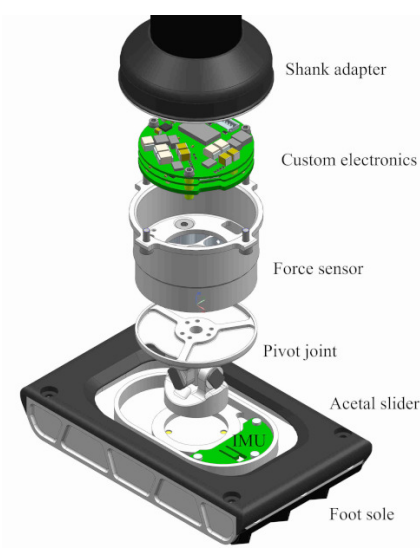

Fig. 2: Sub-assemblies of the foot

We designed sensor-equipped, adaptive feet to enhance locomotion on rough and slippery terrains while measuring local ground inclination and superficial properties. Similarly to ANYmal, the feet have to be sufficiently robust to operate continuously in a challenging environment while while bearing a load of $200 \mathrm{~N}$ (determined from data acquired on the ANYmal). The design consists of a large flat contact surface that can comply to the local ground inclination without interfering with the kinematics of the leg.

We considered a possible inclination of the terrain of up to $25^{\circ}$. The range of motion (ROM) for ground compliance is set to $50^{\circ}$ for the pitch- and $30^{\circ}$ for the roll axis. Since each leg only allows for hip abduction/adduction, hip flexion/extension, and knee flexion/extension, the foot compliance around yaw prevents slipping while turning. With a weight of $314 \mathrm{~g}$ (including cabling and connectors), it is lighter than the original point foot. Fig. 2 illustrates the sub-assemblies of the foot, which the following paragraphs describe in details.

1) Foot sole: The sole has a surface area of $60 \mathrm{~cm}^{2}(100 \mathrm{~mm}$ $\mathrm{x} 60 \mathrm{~mm}$ ) and is made from an off-road rubber tire featuring $5 \mathrm{~mm}$ studs for increased traction (Fig.1b). The sole is connected to a metal rim by clamping, which avoids peeling and gluing issues. A damping foam placed between the rubber sole and the metal structure reduces the peak loads resulting from impact forces during walking. An acetal slider avoids the foot getting stuck on overhanging edges and retains the metal rim.

2) Pivot joint: The pivot joint features a lightweight, universal joint with integrated end stops to provide the required ground compliance. An Ester Polyurethane rubber tube of Shore A70 surrounds the universal joint, providing the retaining force to reset the foot to its initial position after a deflection.

3) Force sensor: A custom, in-house developed 6-axis force/torque sensor ${ }^{1}$ is placed above the pivot joint to measure the forces acting on the foot. It consists of a force sensing element with strain gauges. The sensor is lightweight and robust and allows sensing up to $1000 \mathrm{~N}$ along the $\mathrm{z}$-axis and $400 \mathrm{~N}$ along the $\mathrm{x}$ and $\mathrm{y}$-axis. The maximum torque the sensor can sense is as $10 \mathrm{Nm}$. The accuracy lies within $1.5 \%$ of the measured value, while the repeatability lies below $0.05 \%$. The sensor is temperature compensated to minimize drift during operation.

4) Custom electronics: The electronics of the foot consists of two IMUs (MPU-9250), a force sensor and a microcontroller board. One IMU is located in the sole, while the PCB in the shank includes the second. Serial peripheral interface bus (SPI) connects both the IMUs and the force sensor to the microcontroller. The IMUs are read out at $1 \mathrm{kHz}$, while force measurements have an update frequency of $400 \mathrm{~Hz}$. The microcontroller board is connected to the robot via EtherCAT and powered through the auxiliary $12 \mathrm{~V}$ power line. The custom 6-axis force/torque features a PCB with analog-todigital converters (ADCs) and a microcontroller that processes the analog signals of the strain gauges. The high-level side records sensor data at $400 \mathrm{~Hz}$.

5) Shank: The carbon fiber shank connects the foot to the knee of the robot. The shank is sealed and features a conical slider for protection of the force sensor.

\footnotetext{
${ }^{1}$ https://www.botasystems.com/
} 
6) Sealing: The joint is protected by thick bellows (visible in Fig.1a), mechanically clamped to the structure and sealed, which improves the ingress protection rating compared to previous work. O-rings and silicone seal all the matching surfaces. Water-proof cable glands and connector protect the cables.

\section{B. Foot pose estimation}

The joint angles of the pivot joint shown in Fig. 2 are not directly measured. To estimate the orientation of the foot, measurements of the IMU in the foot sole are fused with ANYmal's state estimation [28]. The state estimation of the robot base relies on points that are stationary w.r.t. the world frame. This would normally be the contact location of a pointfoot, but in our case to rotational center of the ankle joint is used. This makes the estimated state of the rest of the robot independent of the estimated foot pose.

The relevant reference frames for the foot pose estimation algorithm are shown in Fig. 3. The body-fixed shank frame $S$, ankle frame $A$, and foot frame $F$ are separated by consecutive rotations around the y-axis and x-axis. The world frame $W$ is the world frame estimated by ANYmal's state estimation, and the frame $W^{\prime}$ has a relative rotation around the z-axis. The goal of the foot pose estimation algorithm shown in Fig. 4 is to estimate the foot pose in the world frame parameterized by the unit quaternion $\mathbf{q}_{W F}$ from IMU angular velocity and acceleration measurements $\left\{{ }_{F} \hat{\boldsymbol{\omega}}_{W^{\prime} F},{ }_{F} \hat{\mathbf{a}}\right\}$ and the estimated shank orientation $\mathbf{q}_{S W}$.

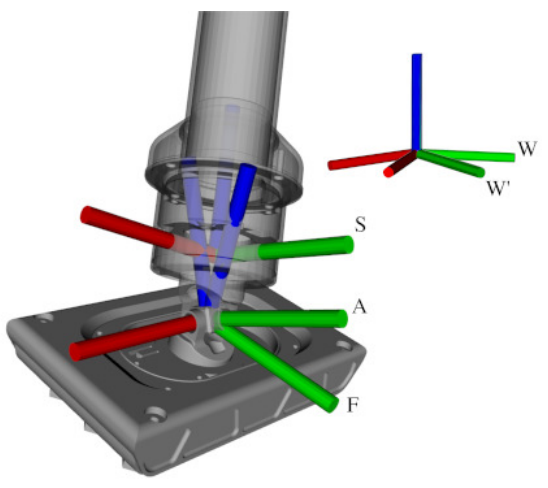

Fig. 3: The reference frames used in the foot pose estimation. The shank frame $S$, ankle $A$, and foot frame $F$ are attached to the corresponding bodies. The frames $W$ and $W^{\prime}$ are world frames with a relative yaw angle in between.

1) Complementary filter: A complementary filter is used to estimate the roll and pitch of the foot in the world frame $W^{\prime}$. First, the gyro bias is estimated by lowpass filtering the angular velocity measurements when the foot is in contact,

$$
{ }_{F} \mathbf{b}_{\boldsymbol{\omega}}^{k+1}=\left(1-\alpha_{\mathbf{b}}\right)_{F} \mathbf{b}_{\boldsymbol{\omega}}^{k}+\alpha_{\mathbf{b} F} \hat{\boldsymbol{\omega}}_{W^{\prime} F} .
$$

Afterwards, the bias corrected angular velocity measurements are used to forward integrate the orientation estimate.

Acceleration measurements are used if the magnitude is close to $1 \mathrm{~g}$, i.e. $\left|\left\|_{F} \hat{\mathbf{a}}\right\|-1\right| \leq \beta_{\mathbf{a}}$. The forward propagated estimate of the foot orientation is used to rotate the measured acceleration ${ }_{F} \hat{\mathbf{a}}$, to the estimated world frame ${ }_{W} \hat{\mathbf{a}}$. The rotational difference between this measured acceleration and the gravity vector $[0,0,1]^{T}$ is given by the quaternion $\Delta \mathbf{q}_{\text {acc }}$.

The correction quaternion is computed as a spherical linear interpolation (SLERP) between $\Delta \mathbf{q}_{\text {acc }}$ and $\mathbf{q}_{I}=[1,0,0,0]^{T}$ with interpolation coefficient $\alpha_{\mathbf{a}}$

$$
\Delta \mathbf{q}_{\text {corr }}=\operatorname{SLERP}\left(\mathbf{q}_{I}, \Delta \mathbf{q}_{\text {acc }} ; \alpha_{\mathbf{a}}\right)
$$

Due to the fundamentally different nature of the swing and contact phase, we use two sets of parameters, shown in Table I. During the stance phase, the gyro bias is estimated, and the estimation relies mostly on acceleration measurements. During the swing phase, the acceleration measurements are less informative due to the motion of the feet, and the filter relies more on the integration of angular velocity.

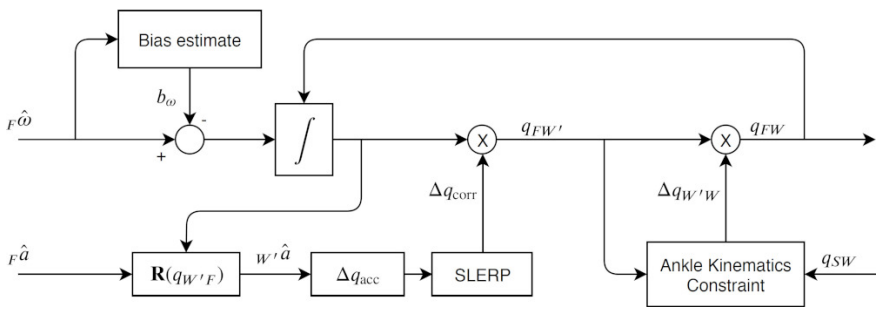

Fig. 4: Foot pose estimation block diagram consisting of a standard complementary filter and a drift compensation using the robot state and ankle kinematics.

2) Drift correction: The drift of the foot orientation $\mathbf{q}_{F W^{\prime}}$ with respect to the world frame used by the robot is expressed as

$$
\mathbf{q}_{F W}=\mathbf{q}_{F W^{\prime}} \otimes \Delta \mathbf{q}_{W^{\prime} W}
$$

where $\Delta \mathbf{q}_{W^{\prime} W}$ is a pure rotation around the z-axis.

The kinematics of the ankle defined as

$$
\mathbf{q}_{F W}=\mathbf{q}_{F A} \otimes \mathbf{q}_{A S} \otimes \mathbf{q}_{S W},
$$

are used to correct for the drift.

Equating both expressions provides the constraint

$$
\begin{aligned}
\mathbf{q}_{F A} \otimes \mathbf{q}_{A S} \otimes \mathbf{q}_{S W} & =\mathbf{q}_{F W^{\prime}} \otimes \Delta \mathbf{q}_{W^{\prime} W} \\
\mathbf{q}_{x}(\phi) \otimes \mathbf{q}_{y}(\theta) \otimes \mathbf{q}_{S W} & =\mathbf{q}_{F W^{\prime}} \otimes \mathbf{q}_{z}(\psi),
\end{aligned}
$$

where $\phi, \theta$, and $\psi$ parameterize the pure roll, pitch, and yaw rotations. For non-singular orientations, the constraint consists of three independent equations and three unknowns. Solving the constraint symbolically results in two solutions $\{\phi, \theta, \psi\}$, neglecting additional full rotations. Typically, only one solution has ankle pitch and roll angles that are within kinematic limits. The corresponding yaw angle is then used to correct for the drift between the two world frames. When two solutions are within the kinematic limits, the drift is not corrected.

\section{Controller}

We use the motion optimization described in [29] to obtain desired torso and feet accelerations based on user commanded 
TABLE I: Foot pose estimation parameters in stance and swing phase.

\begin{tabular}{|l|c|c|c|}
\hline Parameters & Symbol & Stance & Swing \\
\hline Angular velocity bias gain & $\alpha_{\mathbf{b}}$ & 0.001 & 0.0 \\
Acceleration correction SLERP coefficient & $\alpha_{\mathbf{a}}$ & 0.02 & 0.005 \\
Acceleration measurement threshold & $\beta_{\mathbf{a}}$ & 0.1 & 0.1 \\
\hline
\end{tabular}

TABLE II: Prioritized tasks used in the hierarchical wholebody controller together with the task's dimensionality and type (equality/inequality).

\begin{tabular}{|c|l|c|c|}
\hline Priority & Task & Dim. & Type \\
\hline 0 & Floating base equations of motion. & 6 & Eq. \\
& Torque limits. & 12 & Ineq. \\
& Friction cone constraint. & $5 n_{c}$ & Ineq. \\
& No motion at the contact points. & $3 n_{c}$ & Eq. \\
\hline \multirow{2}{*}{1} & Center of mass linear motion tracking. & 3 & Eq. \\
& Base angular motion tracking. & 3 & Eq. \\
& Swing leg position tracking. & $12-3 n_{c}$ & Eq. \\
\hline 2 & Contact force optimization. & $3 n_{c}$ & Eq. \\
\hline
\end{tabular}

torso velocities and a desired gait pattern. Estimation of the contact state is described in [30], along with reactive measures when the desired and measured state differ. In short, a probabilistic algorithm fuses dynamic and kinematic information to classify a foot to be in contact, swing, or slip. When a leg is classified to be in swing, but a contact was desired, a vertical motion is initiated to establish contact. In case of a slip event, joint impedance gains are increased to stabilize the leg.

In this work, we adapt the tracking controller to exploit the information about local ground inclination. We use the hierarchical whole-body control approach described in [27], which optimizes over generalized accelerations $\dot{\mathbf{u}} \in \mathbb{R}^{n_{u}}$ and $\boldsymbol{\lambda} \in \mathbb{R}^{3 n_{c}}$, where $n_{u}$ is the number of generalized velocities, and $n_{c}$ is the number of contact points. Because of the low joint stiffness and small footprint of the foot compared to the full support polygon, each foot is modelled as a single contact point located at the center of rotation of the ankle joint. The list of prioritized tasks is given in Table II together with the amount and type of constraints they impose. The number of independent dimensions left after applying the physical constraints at priority 0 , and motion tracking constraints at priority 1 , has been extensively studied in [31].

Internal forces, which do not influence the robot's net acceleration, can be realized between contact points when the system is an overconstrained situation. While [31] outlines the mathematical details, we provide an intuitive explanation in the following paragraph.

In full stance phase, twelve independent contact force directions exist ( 3 contact constraints per leg), with only six un-actuated base coordinates to control. There are, therefore, six dimensions left to optimize during the contact optimization in priority 2 . With three, and two contact points, the remaining dimensionality reduces to three and one respectively. For a single and no contact points, there are no internal forces that can be created.

The reference controller, referred to as standard controller, estimates a single terrain normal by fitting a plane through all available contact points. This estimated plane provides the surface normal for the friction cone constraint.
For the Terrain aware controller presented in this work, the friction cone constraint and contact force optimization tasks are adapted to incorporate the information about local ground inclination. For the other tasks, we refer the reader to [27]. From each estimated foot orientation, a surface normal, ${ }_{W} \mathbf{n}_{i}$, and a tangential basis ${ }_{W} \mathbf{T}_{i}$ are extracted. The tangential basis, $\mathbf{T}_{i}=\left[\mathbf{t}_{i, h}, \mathbf{t}_{i, l}\right]$, contains two unit vectors, where ${ }_{W} \mathbf{t}_{i, h}$ is aligned with the heading of the torso. The contact forces corresponding to the i-th foot are denoted by ${ }_{W} \boldsymbol{\lambda}_{i}$. In the following sections, the subscript indicating the world frame is dropped to simplify the notation.

1) Friction cone constraint: The friction cone constraint is implemented as a friction pyramid, for each foot

$$
\begin{aligned}
\left( \pm \mathbf{t}_{i, h}-\mu \mathbf{n}_{i}\right)^{T} \boldsymbol{\lambda}_{i} & \leq 0 \\
\left( \pm \mathbf{t}_{i, l}-\mu \mathbf{n}_{i}\right)^{T} \boldsymbol{\lambda}_{i} & \leq 0 \\
-\mathbf{n}_{i}^{T} \boldsymbol{\lambda}_{i} & \leq-\lambda_{\min }
\end{aligned}
$$

where $\mu$ is the friction coefficient, and $\lambda_{\min }$ is a minimal contact force to avoid loss of contact. These parameters are set to 0.5 and $5 \mathrm{~N}$ respectively and remain fixed for all experiments. Note that due to the approximation of the cone as a pyramid, a ratio of tangential and normal forces up to $0.5 \sqrt{2}$ is possible.

2) Contact force optimization: On flat ground, the lowest priority task is typically used to either minimize torques or internal forces. Minimizing internal forces can be achieved by a contact force optimization task that minimizes the magnitude of the contact forces $\left\|\boldsymbol{\lambda}_{i}\right\|_{2}^{2}$. On inclined surfaces, however, it becomes desirable to create internal forces such that the resulting contact forces are aligned with the local surface normal. To that end, we formulate the following optimization,

$$
\min _{\boldsymbol{\lambda}} \sum_{i=1}^{n_{c}}\left\|\mathbf{T}_{i}^{T} \boldsymbol{\lambda}_{i}\right\|_{2}^{2}+\gamma^{2}\left(\mathbf{n}_{i}^{T} \boldsymbol{\lambda}_{i}\right)^{2},
$$

where $\gamma$ is a regularization parameter. Note that $\gamma=1.0$ retrieves the task that minimizes contact forces. Choosing $\gamma=0.0$ leads to undesirable effects when the normal vectors of all feet are close to parallel in full stance configuration. In that case, the optimization problem in the last task becomes highly sensitive to small deviations in the surface normals, resulting in an unequal force distribution between the feet and an increased sensitivity to noise. Take the example of the robot standing on four feet on flat ground with vertical surface normal. If one foot has a minimal tilt angle, the optimal solution is to completely unload this leg while standing on the other three. Penalizing the force magnitude in normal direction regularizes these cases by promoting an equal force distribution between the legs.

To fit the hierarchical whole-body control framework, the minimization in (10) is transcribed into an equality task,

$$
\left[\begin{array}{ll}
\mathbf{0}_{3 n_{c} \times n_{u}} & \text { blockdiagonal }
\end{array}\left(\left[\begin{array}{c}
\mathbf{T}_{i}^{T} \\
\gamma \mathbf{n}_{i}^{T}
\end{array}\right]\right)\right]\left[\begin{array}{c}
\dot{\mathbf{u}} \\
\boldsymbol{\lambda}
\end{array}\right]=\mathbf{0} .
$$

\section{RESULTS AND DISCUSSION}

\section{A. Test setup}

ANYmal, a dog-sized quadrupedal robot, has been used as testing platform because it provides the torque controllability 


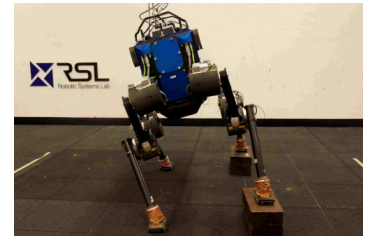

(a) Blocks

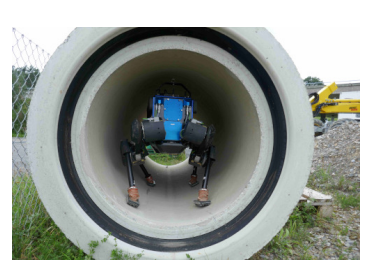

(c) Concrete sewer tube

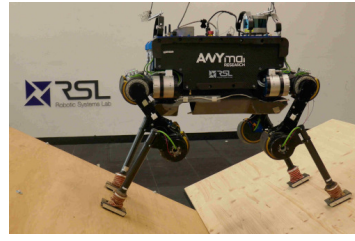

(b) Adjustable V-shaped terrain

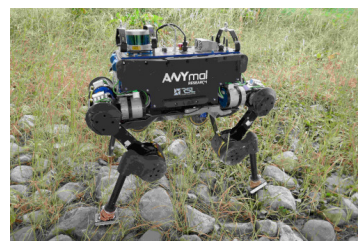

(d) Large round stones
Fig. 5: Terrains used for testing.

[7] required by the controller presented in II-C. We validated the proposed system with a bottom-up approach: first, the sensing capabilities of the feet have been confirmed (III-B), then the performances of the terrain aware controller have been measured (III-C), and finally the effects on locomotion were evaluated (III-D). All the test were performed with a human operator giving velocity commands to the robot.

Terrains and obstacles with pronounced differences between local and average inclination have been selected to highlight the sensing capabilities of the sensorized feet and the tangential force minimization of the controller. We evaluated the impact on locomotion performance with outdoor tests on terrains where slippage would occur easily in case of poor contact forces distribution. The complete selection of terrains consists in:

- blocks with a height of $15 \mathrm{~cm}$ placed under the left feet, Fig.5a;

- inclined wood planks, with adjustable slope, assembled in V shape, Fig.5b;

- round concrete sewer tube, Fig.5c;

- round large stones fixed to the ground, Fig.5d.

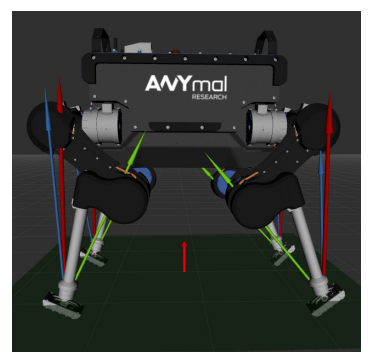

(a) Standard controller

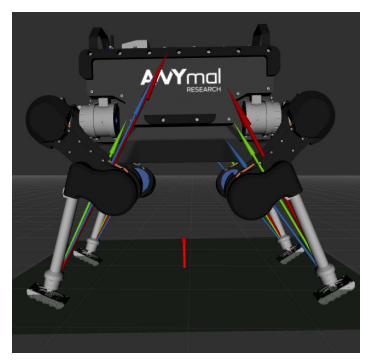

(b) Terrain aware controller
Fig. 6: 3D visualization of ANYmal. Surface normals are represented in green, desired contact forces in red, measured contact forces in blue and interpolated surface normal in bright red.

Fig.6 consists of two visualizations of ANYmal, with representations of the vectors used in the following analysis. Surface normals measured by the sensorized feet, are represented by green arrows. The plane interpolated on the contact points is represented as a shaded rectangle and its estimated normal, named $\tilde{\mathbf{n}}$, is represented by the bright red small arrow. To better measure the difference between the actual terrain normal and those measured, the error angle $\alpha_{e}$ is introduced, defined as the angle between the estimated normal and the actual one.

The feet contact forces are named $\mathbf{F}^{\mathbf{i}}$, which in the analysis are decomposed in components perpendicular and tangential to the contact surface, respectively $\mathbf{F}_{\perp}^{\mathbf{i}}$ and $\mathbf{F}_{\|}^{\mathbf{i}}$. In Fig. 6 . Red arrows represent the contact forces commanded by the controller, while blue arrows represent the forces measured by the sensorized feet.

\section{B. Sensorized foot sensing capabilities}

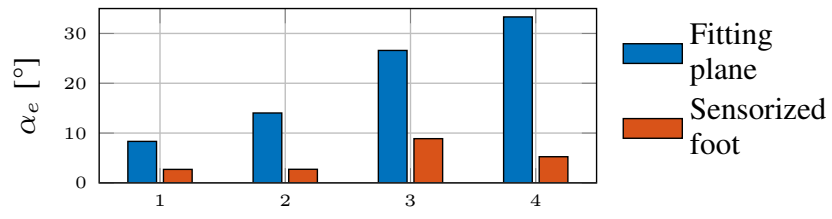

Fig. 7: Maximum error angles over different terrains. Terrains considered:block in pitch and roll directions (1 and 2), Vshaped terrain in pitch and roll direction (3 and 4).

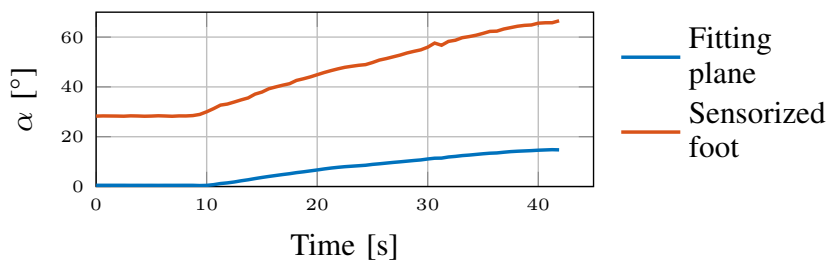

Fig. 8: Surface angles measured by fitting plane and sensorized foot during changing pitch experiment.

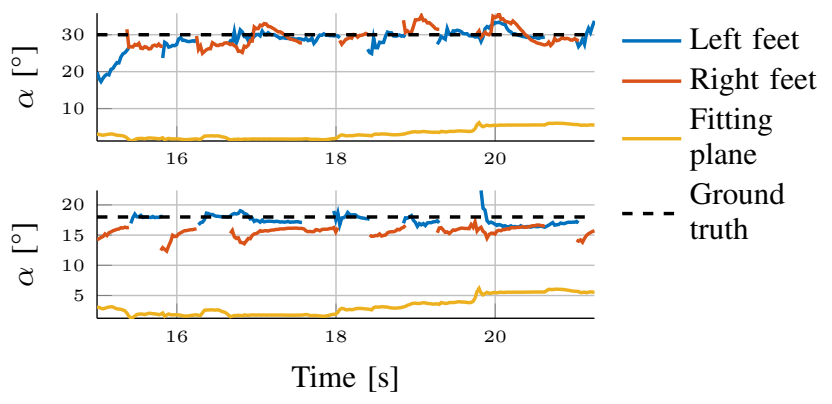

Fig. 9: Surface inclinations measured by sensorized feet and fitting plane while walking over V shaped terrain. Front feet in top graph, hind feet in bottom graph.

Fig.6 shows two visualizations of ANYmal standing over a V-shaped terrain, the qualitative difference between $\mathbf{n}_{i}$ and $\tilde{\mathbf{n}}$ is visible in the representation. The graph in Fig. 7 gives a quantitative representation of the difference. The maximum error angles produced by the fitting plane and by the sensorized feet are shown, respectively in blue and red. The terrains considered for this test consist of the blocks in pitch and 
roll configuration ( 1 and 2 in the graph) and $\mathrm{V}$ terrain with $30^{\circ}$ slope in roll and pitch (3 and 4). The difference is greater for terrain characterized by large local inclinations and where the feet are all at the same height. In such cases, the error angle for $\tilde{\mathbf{n}}$ almost coincide with the local inclination, while the sensorized foot can produce errors significantly smaller.

The graph in Fig. 8 shows the surface inclination measured by the fitting plane and the sensorized foot during a quasi-static experiment, where we progressively increased the inclination of one of the side of the $\mathrm{V}$ terrain until slippage occurred. The sensorized foot is more sensitive to the changing inclination, while the fitting plane is only marginally affected. We performed the experiment using the terrain aware controller. Such angle would be otherwise infeasible with the standard controller as shown in III-C

The graph in Fig. 9 shows the performance of the sensorized foot while trotting on the $\mathrm{V}$ terrain. The upper graph represents the front feet, while the bottom represents the hind ones. The absolute surface inclination is calculated using the normals from the complementary filter described in II-B. The ground truth inclinations, measured separately, are also plotted in the graph. According to the data, the normal estimation from the sensorized foot outperforms the fitting plane also while walking. A higher level of noise in the feet data is present, which can be attributed to impacts of the other feet during locomotion.

\section{Tangential force optimization}

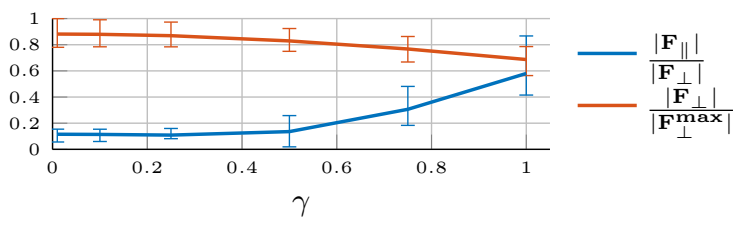

Fig. 10: The average tangential to normal force ratio as a function of the regularization parameter $\gamma$ in (10). Force ratios are measured for a fixed configuration with ANYmal standing on the V-shaped terrain. The error bars represent the maximum and minimum values across the feet.

We investigated the effect of the regularization parameter $\gamma$ in (10) on ANYmal standing on the $\mathrm{V}$ terrain with surface inclinations of 30 and 19 degrees. Fig. 10 summarizes the results of the test, where the ratio between measured tangential and normal forces is plotted for different values of $\gamma$. Additionally, the ratio between the normal forces and the maximum across the experiments is provided. As expected, the maximum normal forces occur for the smallest value of $\gamma$. The trade-off driven by $\gamma$ is visible: for large values, the contact force magnitude is minimized at the expenses of more significant tangential components, while for a smaller value the tangential components are reduced by creating additional internal forces.

A value of $\gamma=0.25$ was selected for the rest of the experiments. Lower values do not significantly decrease the magnitude of tangential forces and start to show increased sensitivity to noise in the normal estimate, as discussed in Section II-C.

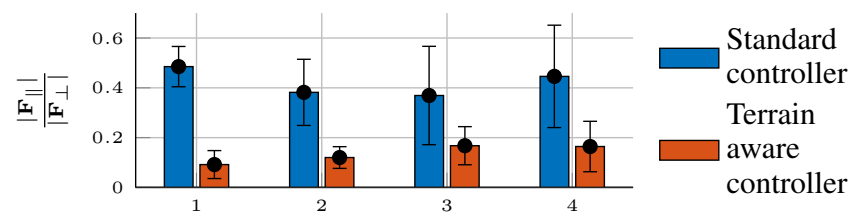

Fig. 11: Average tangential to perpendicular force ratio for the two control strategies. Terrains used: V-shaped terrain in pitch and roll direction (1 and 2), sewer tube (3) and large stones (4)

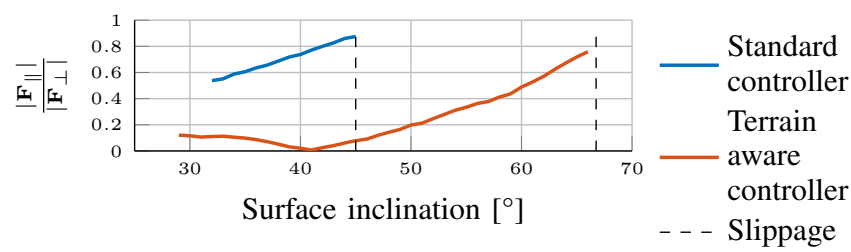

Fig. 12: Tangential to normal force ratio for for variable pitch experiment.

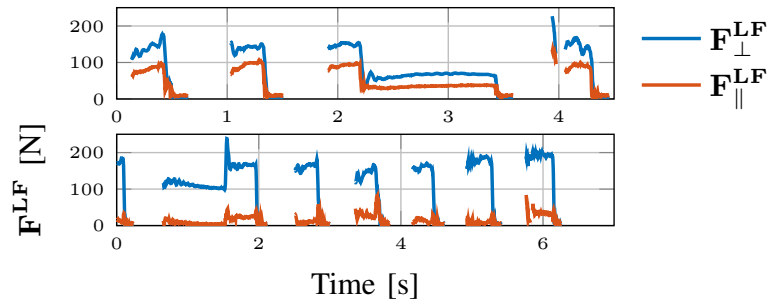

Fig. 13: Normal and absolute tangential components of the contact forces while walking on V-shaped terrain terrain. Standard controller on top and terrain aware controller on bottom.

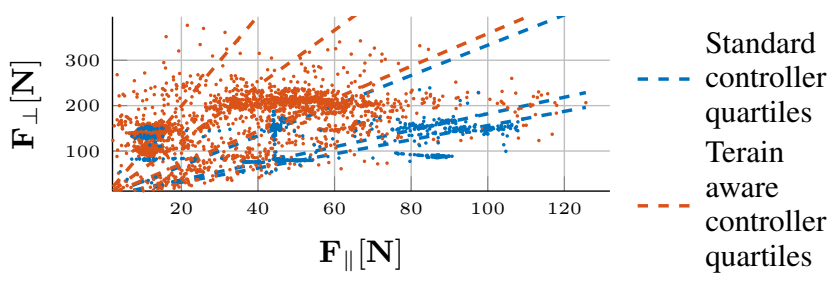

Fig. 14: Scatter plot of normal and tangential force components while walking on V-shaped terrain terrain.

In Fig. 6, the qualitative difference between the standard and the terrain aware controller is evident. The contact forces, both the requested and the measured, are vertical for the standard controller and roughly aligned to the local ground normal for the terrain aware controller. Fig. 11 gives a quantitative counterpart. The graph shows the behavior of the tangential to normal force ratio during different experiments. We conducted these experiments in stationary conditions on the $\mathrm{V}$-shaped terrain (pitch and roll direction, respectively 1 and 2 in the 
plot), in the sewer tube (3) and in over the large stones (4). The black error bars show the maximum and minimum ratio measured. The experiments performed over different terrains confirm that the controller is effective in reducing the tangential component of the contact forces.

The graph in Fig. 12 illustrates the tangential to normal contact force ratio for the same quasi-static experiment of Fig. 8. The experiment shows how the terrain aware controller always manage to maintain a lower ratio (for the same local inclination) and how this results in a higher maximum inclination before slippage, which occurs roughly at the same ratio. The asymmetry of the terrain explains the non-monotonous behavior and the local minimum occurs when opposing surfaces have exactly the same inclination.

The graph in Fig. 13 shows the normal and absolute tangential component of the left foot contact force while walking over the V-shaped terrain with the standard and the terrain aware controller. The graphs show qualitatively that the proposed controller reduces the magnitude of the tangential component also when walking and not only in static conditions. The scatter plots in Fig. 14 gives a more complete picture of $\mathbf{F}_{\perp}^{\mathbf{i}}$ and $\mathbf{F}_{\|}^{\mathbf{i}}$ for the same experiment. The forces recorded during the experiment are represented as dots, while the dashed lines represent the quartiles of the distribution. In the scatter plot the number of points has been reduced for clarity. It is evident how the terrain aware controller moves the distribution towards lower tangential to normal ratio.

\section{Effect on locomotion}

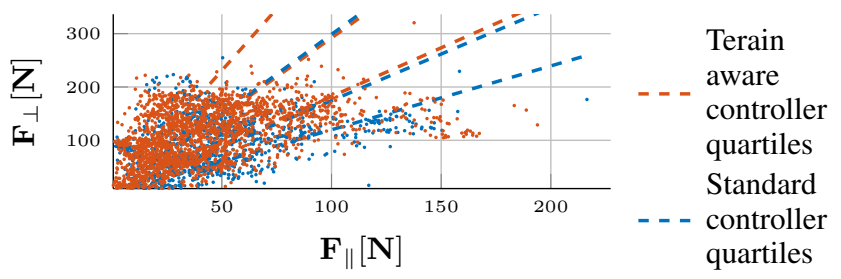

Fig. 15: Scatter plot of forces while walking on large round stones.

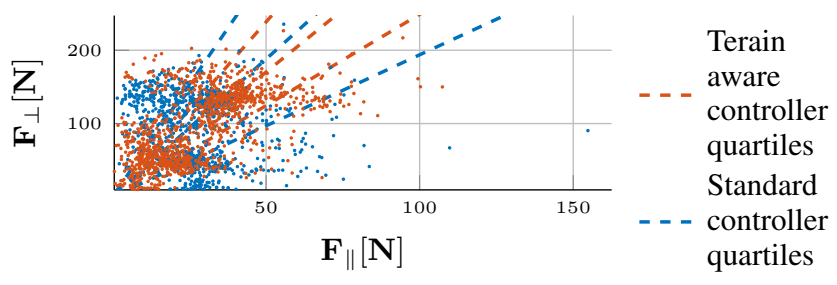

Fig. 16: Scatter plot of forces while walking inside the tube.

Fig.15 and 16 show a scatter plots of the contact force while walking on the large stones of picture $5 \mathrm{~d}$ and in the pipe of Fig. 5c. While the shift towards lower ratio observed in 14 is confirmed, the effect is less significant than in the lab tests. This reduced effect is a prevalent result also for other terrains. One possible interpretation for this effect could be the shape of the terrain less suitable for producing internal forces

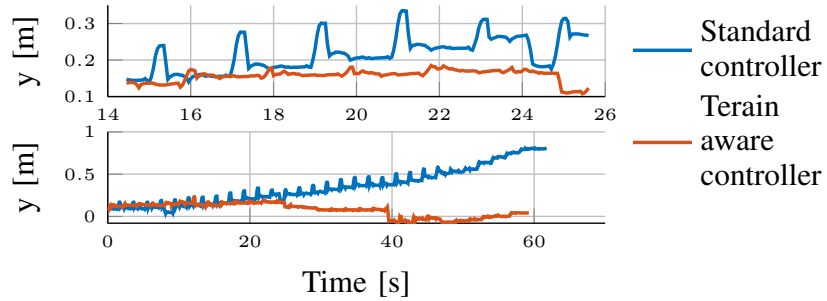

Fig. 17: LF foot y coordinate while walking inside concrete tube.

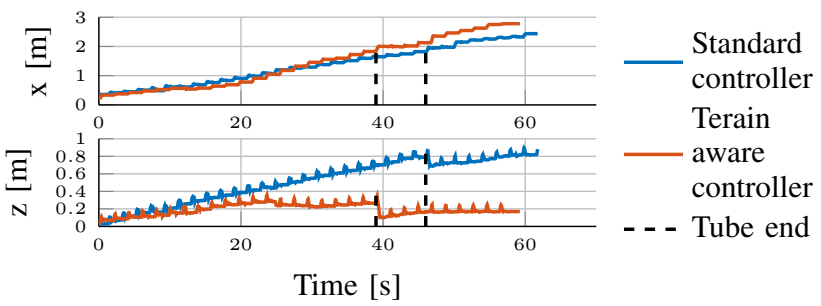

Fig. 18: $\mathrm{LF}$ foot $\mathrm{x}$ and $\mathrm{z}$ coordinates while walking inside concrete tube.

(compared to the V-shaped terrain). Another effect is slippage which occurs with both controllers, however with different intensity, and interferes with the force distribution.

Fig.17 show the behavior of the y coordinate of the left front foot while walking in the pipe. The top graph focuses on the short period dynamics: the standard controller introduces very evident spikes of $10 \mathrm{~cm}$ completely absent in the terrain aware controller. Slippage, clearly observed during the tests, explain the spikes. The foothold locations commanded by the controller are too steep. Immediately after touchdown, the foot slips toward the center of the pipe. The bottom graph shows the long period effect of slippage: after only 50 seconds, the time necessary to traverse the $3 \mathrm{~m}$ tube, the state estimator drifted over $0.5 \mathrm{~m}$. The drift for the terrain aware controller instead is not visible from the graph. Fig. 18 shows the behavior of the $\mathrm{x}$ (top) and $\mathrm{z}$ (bottom) coordinates. From the $\mathrm{x}$ plot, a slightly higher velocity can be attributed to the terrain aware controller which reaches the end of the tube before the standard controller. The $\mathrm{z}$ graph shows again a significant drift for the standard controller and a much more contained drift for the terrain aware controller.

It can be concluded that even if the effect on the optimization of the tangential force is smaller than in the artificial test case with very defined geometry, the terrain aware controller has a positive effect on locomotion by reducing slippage and containing drift of the state estimator.

\section{Conclusion}

In this paper, a sensorized foot design, capable of measuring the ground contact forces as well as the ground normal using force/torque and IMU sensors, was presented, together with a control strategy that leverages its capabilities by optimization of the contact forces. The experiments confirmed an improvement in the perception capabilities of ANYmal, which is now able to sense the inclination of the ground at the contact 
points and locally measure the ground reaction force. The additional information can be effectively used to minimize the tangential component of the contact forces, which result in a lower probability of slipping. The improved force distribution is particularly effective for terrains characterized by strong local inclination and low friction coefficient.

While the results obtained so far are encouraging, the proposed approach could be further improved both on the hardware and on the software side. Replacing the IMUs with encoders could reduce the amount of noise introduced by impact and vibrations. A fusion of the information coming from the feet in the state estimation could lead to a more performant and reliable estimation of the contact state, with consequent benefits.

\section{ACKNOWLEDGMENT}

The authors want to thank Klajd Lika and Ilias Patsiaouras from Bota Systems as well as Markus Stäuble from ETH - RSL for their help in integrating the force/torque sensors into the feet.

\section{REFERENCES}

[1] Jörg P. Müller. Control architectures for autonomous and interacting agents: A survey. In Lawrence Cavedon, Anand Rao, and Wayne Wobcke, editors, Intelligent Agent Systems Theoretical and Practical Issues, pages 1-26, Berlin, Heidelberg, 1997. Springer Berlin Heidelberg.

[2] Masato Hirose and Kenichi Ogawa. Honda humanoid robots development. Philosophical Transactions of the Royal Society A: Mathematical, Physical and Engineering Sciences, 365(1850):11-19, 2007.

[3] Thomas Buschmann, Sebastian Lohmeier, and Heinz Ulbrich. Humanoid robot lola: Design and walking control. Journal of Physiology-Paris, 103(3):141 - 148, 2009. Neurorobotics.

[4] Ill-Woo Park, Jung-Yup Kim, Jungho Lee, and Jun-Ho Oh. Mechanical design of humanoid robot platform khr-3 (kaist humanoid robot 3: Hubo). pages $321-326,022005$.

[5] F. Negrello, M. Garabini, M. G. Catalano, P. Kryczka, W. Choi, D. G. Caldwell, A. Bicchi, and N. G. Tsagarakis. Walk-man humanoid lower body design optimization for enhanced physical performance. In 2016 IEEE International Conference on Robotics and Automation (ICRA), pages 1817-1824, May 2016.

[6] C. Semini, V. Barasuol, J. Goldsmith, M. Frigerio, M. Focchi, Y. Gao, and D. G. Caldwell. Design of the hydraulically actuated, torquecontrolled quadruped robot hyq2max. IEEE/ASME Transactions on Mechatronics, 22(2):635-646, April 2017.

[7] M. Hutter, C. Gehring, A. Lauber, F. Gunther, C. D. Bellicoso, V. Tsounis, P. Fankhauser, R. Diethelm, S. Bachmann, M. Bloesch, H. Kolvenbach, M. Bjelonic, L. Isler, and K. Meyer. Anymal - toward legged robots for harsh environments. Advanced Robotics, 31(17):918 931, 2017.

[8] Gerardo Bledt, Matthew J Powell, Benjamin Katz, Jared Di Carlo, Patrick Wensing, and Sangbae Kim. Mit cheetah 3: Design and control of a robust, dynamic quadruped robot. 102018.

[9] B. Katz, J. D. Carlo, and S. Kim. Mini cheetah: A platform for pushing the limits of dynamic quadruped control. In 2019 International Conference on Robotics and Automation (ICRA), pages 6295-6301, May 2019.

[10] S. Hirose, K. Yoneda, and H. Tsukagoshi. Titan vii: quadruped walking and manipulating robot on a steep slope. In Proceedings of International Conference on Robotics and Automation, volume 1, pages 494-500 vol.1, April 1997.

[11] S. Abad, N. Herzig, S. M. H. Sadati, and T. Nanayakkara. Significance of the compliance of the joints on the dynamic slip resistance of a bioinspired hoof. IEEE Transactions on Robotics, pages 1-14, 2019.

[12] Hector Montes, Samir Nabulsi, and Manuel A. Armada. Reliable, builtin, high-accuracy force sensing for legged robots. The International Journal of Robotics Research, 25(9):931-950, 2006.
[13] K. Suwanratchatamanee, M. Matsumoto, and S. Hashimoto. Haptic sensing foot system for humanoid robot and ground recognition with one-leg balance. IEEE Transactions on Industrial Electronics, 58(8):3174-3186, Aug 2011.

[14] J. Rogelio Guadarrama-Olvera, F. Bergner, E. Dean, and G. Cheng. Enhancing biped locomotion on unknown terrain using tactile feedback. In 2018 IEEE-RAS 18th International Conference on Humanoid Robots (Humanoids), pages 1-9, Nov 2018.

[15] K. Fondahl, D. Kuehn, F. Beinersdorf, F. Bernhard, F. Grimminger, M. Schilling, T. Stark, and F. Kirchner. An adaptive sensor foot for a bipedal and quadrupedal robot. In 2012 4th IEEE RAS EMBS International Conference on Biomedical Robotics and Biomechatronics (BioRob), pages 270-275, June 2012.

[16] L WAGNER, Péter Fankhauser, Michael Bloesch, and Marco Hutter Foot contact estimation for legged robots in rough terrain: Proceedings of the 19th International Conference on CLAWAR 2016, pages 395-403. 102016.

[17] Shigeo Hirose, Souichi Inoue, and Kan Yoneda. The whisker sensor and the transmission of multiple sensor signals. Advanced Robotics, 4(2):105-117, 1989.

[18] Marco Hutter, Mark Hoepflinger, C Remy, and Roland Siegwart. Hybrid operational space control for compliant legged systems. 072012.

[19] Michele Focchi, Andrea del Prete, Ioannis Havoutis, Darwin G. Featherstone, Roya nd Caldwell, and Claudio Semini. High-slope terrain locomotion for torque-controlled quadruped robots. Autonomous Robots, 41(1):259-272, Jan 2017.

[20] Ludovic Righetti et al. Optimal distribution of contact forces with inverse-dynamics control. The International Journal of Robotics Research, 32(3):280-298, 2013.

[21] C. Gehring et al. Dynamic trotting on slopes for quadrupedal robots. In 2015 IEEE/RSJ International Conference on Intelligent Robots and Systems (IROS), pages 5129-5135, Sep. 2015.

[22] C. Dario Bellicoso et al. Perception-less terrain adaptation through whole body control and hierarchical optimization. In 2016 IEEE-RAS 16th International Conference on Humanoid Robots (Humanoids), pages 558-564, Nov 2016.

[23] Peter D. Neuhaus, Jerry Pratt, and Matthew Johnson. Comprehensive summary of the institute for human and machine cognition's experience with littledog. I. J. Robotic Res., 30:216-235, 012011.

[24] Matt Zucker et al. Optimization and learning for rough terrain legged locomotion. The International Journal of Robotics Research, 30(2):175191, 2011.

[25] Péter Fankhauser, Marko Bjelonic, Dario Bellicoso, Takahiro Miki, and Marco Hutter. Robust rough-terrain locomotion with a quadrupedal robot. 052018.

[26] Michele Focchi, Romeo Orsolino, Marco Camurri, Victor Barasuol, Carlos Mastalli, Darwin Caldwell, and Claudio Semini. Heuristic Planning for Rough Terrain Locomotion in Presence of External Disturbances and Variable Perception Quality. 012018.

[27] C. Dario Bellicoso, F. Jenelten, P. Fankhauser, C. Gehring, J. Hwangbo, and M. Hutter. Dynamic locomotion and whole-body control for quadrupedal robots. In 2017 IEEE/RSJ International Conference on Intelligent Robots and Systems (IROS), pages 3359-3365, Sep. 2017.

[28] M. Bloesch et al. State estimation for legged robots on unstable and slippery terrain. In 2013 IEEE/RSJ International Conference on Intelligent Robots and Systems, pages 6058-6064, Nov 2013.

[29] C Dario Bellicoso, Fabian Jenelten, Christian Gehring, and Marco Hutter. Dynamic locomotion through online nonlinear motion optimization for quadrupedal robots. IEEE Robotics and Automation Letters, 3(3):2261-2268, 2018.

[30] F. Jenelten et al. Dynamic locomotion on slippery ground. IEEE Robotics and Automation Letters, 4(4):4170-4176, 2019.

[31] M Hutter, H Sommer, C Gehring, M. Hoepflinger, M Bloesch, and $\mathrm{R}$ Siegwart. Quadrupedal locomotion using hierarchical operational space control. The International Journal of Robotics Research (IJRR), 33(8):1062-1077, may 2014. 